OPEN ACCESS

Edited by:

Gregoire P. Millet

University of Lausanne, Switzerland

Reviewed by:

Marco Alessandro Minetto,

University of Turin, Italy

Zsolt Radak,

Semmelweis University, Hungary

Giuseppe D'Antona,

University of Pavia, Italy

*Correspondence:

Audrius Snieckus

audrius.snieckus@/su.lt

Specialty section:

This article was submitted to

Exercise Physiology,

a section of the journal

Frontiers in Physiology

Received: 22 February 2017

Accepted: 21 July 2017

Published: 03 August 2017

Citation:

Rutkauskas S, Paleckis V

Skurvydas A, Satkunskiene D,

Brazaitis M, Snieckus A,

Baranauskiene N, Rancevas R and

Kamandulis S (2017) Intratendinous

Air Phenomenon: A New Ultrasound

Marker of Tendon Damage?

Front. Physiol. 8:570.

doi: 10.3389/fphys.2017.00570

\title{
Intratendinous Air Phenomenon: A New Ultrasound Marker of Tendon Damage?
}

\section{Saulius Rutkauskas ${ }^{1}$, Vidas Paleckis ${ }^{1}$, Albertas Skurvydas ${ }^{1}$, Danguole Satkunskiene ${ }^{1}$, Marius Brazaitis ${ }^{1}$, Audrius Snieckus ${ }^{1 *}$, Neringa Baranauskiene ${ }^{1}$, Ruslanas Rancevas ${ }^{2}$ and Sigitas Kamandulis ${ }^{1}$}

${ }^{1}$ Institute of Sports Science and Innovation, Lithuanian Sports University, Kaunas, Lithuania, ${ }^{2}$ Department of Radiology, Lithuanian University of Health Sciences, Kaunas, Lithuania

Purpose: To explore the presence of intratendinous air in physically active males after different types of strenuous physical exercise.

Materials and Methods: To detect foci (air bubbles) in the quadriceps femoris tendon (QFT) and the proximal and distal parts of the patellar tendon, ultrasound examination was performed under two conditions: (1) after high-intensity cycling on a cycle ergometer (metabolic); (2) after 200 drop jumps (exercise-induced muscle damage). Based on the results of these two interventions, the presence of air in the tendons after 100 drop jumps was examined further with frequently repeated ultrasound measurements.

Results: Foci were detected in exercise-induced muscle damage. Twenty-three of Sixty investigated tendons (38.3\%) were observed to contain hyperechoic foci after $100 \mathrm{drop}$ jumps. QFT foci were present in 13/23 cases (56.5\%). The location of foci in the QFT was mostly lateral and centro-lateral (76.9\%). The foci disappeared completely between 40 and 180 min after completing 100 drop jumps.

Conclusions: The presence of intratendinous air seems related to high-magnitude, high-force, high-strain exercise of the particular tendon areas. It might represent the stress response of tendons to overload condition.

Keywords: vacuum phenomenon, exercise-induced muscle damage, metabolic fatigue, drop jumps, tendons

\section{INTRODUCTION}

The vacuum phenomenon is currently defined as the accumulation of air in natural cavities such as synovial joints and intervertebral discs (Jordanov and Block, 2010; D'Anastasi et al., 2011; Motamedi et al., 2014). It has been demonstrated that air can be found in hip (Fairbairn et al., 1995; Liu et al., 2015; Schröder et al., 2016), shoulder (Ito et al., 2008; Jordanov and Block, 2010), sternoclavicular (Ito et al., 2008), metacarpophalangeal (Malghem et al., 2011), temporomandibular (Moncada et al., 2008), and knee joints (Jordanov and Block, 2010) and in degenerative intervertebral discs (Stallenberg et al., 2001; D'Anastasi et al., 2011; Feng et al., 2011; Wadhwa et al., 2016). The features of the vacuum phenomenon detected using different imaging techniques have been discussed widely (Coulier, 2004; Gohil et al., 2014; Yanagawa et al., 2016). Fick was the first to identify the vacuum phenomenon on radiographic images (Fick, 1919). Since then it has been demonstrated that computed tomography (CT) has 
a higher sensitivity than radiography and magnetic resonance imaging (MRI) for evaluating the presence of intra-articular air in large joints and vertebral discs (Ito et al., 2008; Moncada et al., 2008; Jordanov and Block, 2010; D’Anastasi et al., 2011; Motamedi et al., 2014; Wadhwa et al., 2016), while radiography and ultrasonography are more sensitive than CT and MRI for evaluating the presence of air in small joints (Malghem et al., 2011).

On ultrasound, intra-articular air is seen as free-floating bright foci of variable size that persist for no more than 30 min (Malghem et al., 2011). During routine performance of musculoskeletal ultrasound, similar foci were surprisingly discovered in the tendons. These foci were usually present at the insertional sites of the tendon, and had a cloud-like or elongated form. The patients showing this phenomenon were of different ages and had different ultrasound pathologies (e.g. meniscus tear or pre-patellar bursitis) or were without abnormal findings. Notably, these bright spots in the tendon were found in almost all basketballers after a short period of training (unpublished data).

Having considered that the tendons are affected by an increased physical load, we suggested a hypothetical "intratendinous air phenomenon." To explore the conditions under which intratendinous air was detected, we applied several strenuous interventions aimed at inducing different types of neuromuscular adaptation in physically active non-athletes. In addition, we evaluated the localization and dynamic changes of the foci. To our knowledge, this is the first scientific study investigating the phenomenon of air in the tendons.

\section{METHODS}

\section{Subjects}

The subjects were randomly recruited healthy males involved in different recreational activities (such as jogging or cycling) of light to moderate intensity at a frequency of 1-2 times per week. They were requested not to exercise for at least 1 week before the study and do not take any medication or dietary supplement during the entire experimental period. None of the subjects had any history of any musculoskeletal disorders or other disease (such as metabolic, inflammatory) that could potentially affect the lower musculature or leg tendons. Ultrasound examination of the knee joints was performed before study and subjects with any signs of pathology (e.g., effusion or tendinopathy) were excluded. All the subjects were free of medications that could directly or indirectly affect tendons, neither before not during the study. Subjects involved in different interventions did not overlap. The mean age and body mass of subjects in interventions I, II, and III were $27.1 \pm 4.8$ years and $79.9 \pm 5.2 \mathrm{~kg}(n=6), 29.8 \pm 9.3$ years and $81.7 \pm 5.8 \mathrm{~kg}(n=10)$, and $25.4 \pm 5.2$ years and $78.7 \pm 4.6 \mathrm{~kg}$ $(n=10)$, respectively. Before the study commenced, all subjects read and signed a written informed consent that was consistent with the principles outlined in the Declaration of Helsinki. Approval for the study of human participants was received from the Regional Ethics Committee before data collection.

\section{Ultrasound Examination}

The ultrasound examination was performed using a Mindray M7 Diagnostic ultrasound system (Shenzhen Mindray Bio-Medical Electronics Co. Ltd, China) with a linear L14-6Ns transducer (10-12 MHz) by a radiologist with 10 years' experience of ultrasound assessment of the musculoskeletal system. To determine that finding did not depend on ultrasound device used, several subjects were assessed with more sophisticated Toshiba ultrasound equipment (linear transducer PLT1005BT, range 5-14 MHz, basic frequency $10 \mathrm{MHz}$, MSK preset). No significant effect of the device was found on the foci distinguishability. The presence of intratendinous foci was evaluated longitudinally and transversally (Figure 1A) with the usual musculoskeletal imaging preset for the quadriceps femoris tendon (QFT), and the proximal (PPT) and distal (DPT) parts of the patellar tendon. The participant was in a supine position with their knees supported and flexed. The examination was performed at the most appropriate degree of knee flexion (approximately $\left.30-40^{\circ}\right)$ at which the tendon is taut with well-defined margins and is homogeneous. For each subject, we acquired at least one image for each tendon at separate locations. Total of 36 tendons ultrasound assessment in Intervention I (six subjects, two sides per subject, three locations per side, two times), 40 tendons in Intervention II (ten subjects, two sides per subject, two locations per side, four times) and 60 tendons in Intervention III (ten subjects, two sides per subject, three locations per side, 16 times) were performed for each person. This resulted in total of 1,192 images for entire study. The images were stored and

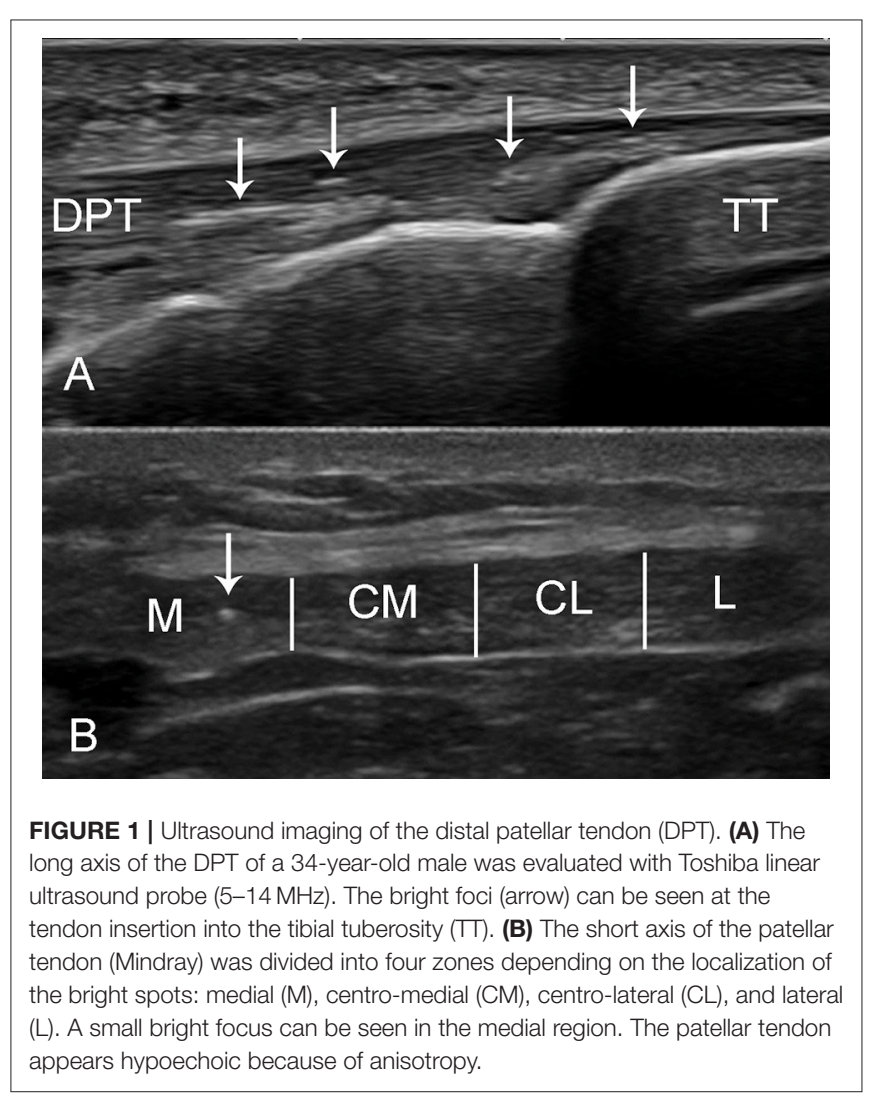


later analyzed independently by two radiologists. The presence of bubbles was confirmed if both radiologists approved at least one separate sub-millimeter-sized focus spot. The localization of the bright spots in each tendon was divided into four zones: medial, centro-medial, centro-lateral, and lateral (Figure 1B).

\section{Study Design}

To test for the presence of foci in tendons, an ultrasound examination was performed under two conditions: (1) after maximum-intensity cycling on a cycle ergometer (extreme metabolic fatigue, intervention I); and (2) after 200 drop jumps (extreme muscle damage, intervention II). Based on the results of these two interventions, the presence of air in the tendons was further examined after 100 drop jumps using frequently repeated ultrasound measurements (intervention III). In addition to the ultrasound measurements, typical markers of exercise-induced muscle damage [muscle strength decline, increased creatine kinase (CK) activity] and metabolic fatigue (performance decline, increased lactate) were assessed. The equipment and techniques used to measure the selected variables were the same as those used in previous studies (Kamandulis et al., 2012).

Intervention I consisted of $6 \times 30 \mathrm{~s}$ bouts of maximumintensity cycling with 4 min rest after each bout; the average power every $5 \mathrm{~s}$ was used for the analyses. The resistance was set at $7.5 \%$ of body weight on a Monark $828 \mathrm{E}$ cycle ergometer (Monark Exercise AB, Sweden). The exercise was preceded by a 10 -min warm-up on a cycle ergometer at a workload of $100 \mathrm{~W}$ and pace of $60 \mathrm{rpm}$. The presence of hyperechoic foci was evaluated after the warm-up and after each cycling bout. Assessment of blood lactate was performed before and immediately after the maximum-intensity cycling.

Intervention II consisted of 200 drop jumps, where the subjects jumped down from a height of $0.5 \mathrm{~m}$, landing on the force platform (Powertimer Testing System, Newtest, Finland) with a countermovement up to $90^{\circ}$ knee angle immediately followed by a maximal jump upward and again landing on the platform. There were 30-s intervals between jumps and 20min intervals after every 50 jumps to minimize changes in the energy metabolites that can affect muscle function. Maximal voluntary contraction (MVC) peak torque of the knee extensor muscles was measured when the subjects were sitting upright in a dynamometer chair (System 3; Biodex Medical Systems, Shiley, New York) with the knee joint positioned at an angle of $110^{\circ}\left(180^{\circ}\right.$ represents full knee extension) for the isometric torque measurements. The peak MVC was maintained for $\sim 3 \mathrm{~s}$ before relaxation and was measured twice; the larger value was used in the analysis. Two additional measurements of MVC were performed with the maximal electrical impulse $(250 \mathrm{~ms}$, $100 \mathrm{~Hz}$; MG 440; Medicor, Budapest, Hungary) superimposed on the voluntary contraction to assess the central activation ratio. Electrical stimuli to the quadriceps muscle were delivered through surface electrode $(9 \times 18 \mathrm{~cm})$ in trains of monopolar square wave pulses of $1 \mathrm{~ms}$ duration (voltage, 120-150 V). The ultrasound examination of the quadriceps tendon and the distal part of the patellar tendon of both legs and the muscle function assessment of the right knee extensor muscles were performed before the intervention, immediately after 100 or 200 jumps and $24 \mathrm{~h}$ after the jumps. Evaluation of CK activity in the blood was performed before and $24 \mathrm{~h}$ after the intervention.

Intervention III consisted of 100 drop jumps performed using the same method and equipment as for intervention II, excluding the MVC and electrical stimulation. There were 30-s intervals between drop jumps and 2-min rest intervals after 2, $4,6,8,10,15,20,25,50,75$, and 100 drop jumps for the ultrasound foci examination. The ultrasound measurements were repeated every $20 \mathrm{~min}$ after completion of 100 drop jumps until the disappearance of the foci.

\section{Statistical Analysis}

Data are presented as the mean $\pm \mathrm{SD}$. A one-way analysis of variance (ANOVA) for repeated measures was used to determine the effects of the intervention on dependent variables. When the dependent variable was not normally distributed, the MannWhitney $U$ or Wilcoxon $W$ test was used to compare groups. The level of significance was set at 0.05 . All calculations were performed using IBM SPSS v. 20 (IBM, Armonk, NJ).

\section{RESULTS}

\section{Intervention I}

Compared with the baseline, the peak power had dropped by 69.7 $\pm 8.1 \%$ at the end of the last cycling bout $(p<0.05)$. The blood lactate concentration increased from $1.5 \pm 0.3 \mathrm{mmol} / \mathrm{L}$ to $19.2 \pm$ $1.4 \mathrm{mmol} / \mathrm{L} 3 \mathrm{~min}$ after completion of the last cycling bout.

Hyperechoic foci were identified in seven of 36 tendons (19.4\%) and usually were observed as separate spots. They were noticed in three of seven DPTs (42.8\%). No foci were observed in any tendon in one of the subjects.

\section{Intervention II}

Declines in MVC of $25.8 \pm 10.2 \%$ and $30.6 \pm 11.6 \%$ were observed after 100 and 200 drop jumps, respectively $(p<0.05$ for both time points). These declines remained significant (25.1 $\pm 8.7 \%, p<0.05) 24 \mathrm{~h}$ after the jumps. Central activation ratio decreased from $97.2 \pm 2.8 \%$ at baseline to $92.4 \pm 7.5 \%$ after 100 drop jumps $(p>0.05)$ and $88.4 \pm 10.1 \%$ after 200 drop jumps ( $p<0.05$, compared to baseline), while remained $90.3 \pm 9.2 \%$ at $24 \mathrm{~h}$ after 200 drop jumps ( $p<0.05$, compared to baseline). The blood CK activity was $153.6 \pm 100.2 \mathrm{IU} \cdot \mathrm{L}^{-1}$ before the jumps and significantly higher $\left(1718.4 \pm 954 \mathrm{IU} \cdot \mathrm{L}^{-1} ; p<0.05\right) 24 \mathrm{~h}$ after the jumps. After 200 jumps, slight changes in the ultrasound were identified in some subjects: one had small effusions on both sides of the deep infrapatellar bursas, and in the another subject increased effusions were noticed in both suprapatellar bursas. After $24 \mathrm{~h}$, all these changes had disappeared.

Intratendinous foci were observed in 21 of 40 tendons (52.5\%, Table 1). Table 2 shows that there was a significant asymmetry of the left and right knees' QFT and DPT: bright spots were observed significantly more frequently in the right knee than the left knee tendons. No foci were observed in any tendon in one of the subjects. 
TABLE 1 | The presence of foci in the quadriceps femoris tendon (QFT), proximal patellar tendon (PPT), and distal patellar tendon (DPT).

\begin{tabular}{|c|c|c|c|c|c|c|c|c|}
\hline \multirow[t]{2}{*}{ Intervention } & \multirow{2}{*}{$\begin{array}{l}\text { Number of } \\
\text { participants }\end{array}$} & \multirow{2}{*}{$\begin{array}{c}\text { Number of } \\
\text { tendons }\end{array}$} & \multicolumn{6}{|c|}{ Appearance of intratendinous air } \\
\hline & & & Right QFT & Right PPT & Right DPT & Left QFT & Left PPT & Left DPT \\
\hline I & 6 & 36 & 1 & 1 & 1 & 1 & 1 & 2 \\
\hline II & 10 & 40 & 8 & - & 7 & 4 & - & 2 \\
\hline III & 10 & 60 & 7 & 1 & 4 & 6 & 1 & 4 \\
\hline Total & 26 & 136 & 16 & 2 & 12 & 11 & 3 & 8 \\
\hline
\end{tabular}

TABLE 2 | The localization of intratendinous air collection according to the different insertional zones of quadriceps femoris tendon (QFT), proximal insertion of patellar tendon (PPT) and distal insertion of patellar tendon (DPT).

\begin{tabular}{|c|c|c|c|c|c|c|c|c|c|}
\hline \multirow[t]{2}{*}{ Tendons } & \multirow[t]{2}{*}{ Experiments } & \multicolumn{4}{|c|}{ Right side } & \multicolumn{4}{|c|}{ Left side } \\
\hline & & Lateral & Centro-later & Centro-medial & Medial & Medial & Centro-medial & Centro-lateral & Lateral \\
\hline \multirow[t]{4}{*}{ QFT } & 1 & - & - & 1 & - & - & - & - & - \\
\hline & 2 & 2 & 7 & 5 & - & - & - & 3 & 1 \\
\hline & 3 & 4 & 6 & 2 & - & - & 1 & 6 & 4 \\
\hline & Total & 6 & 13 & 8 & - & - & 1 & 9 & 5 \\
\hline \multirow[t]{3}{*}{ PPT } & 1 & - & - & 2 & - & - & - & - & \\
\hline & 3 & - & 1 & 1 & 1 & 1 & 1 & - & - \\
\hline & Total & - & 1 & 3 & 1 & 1 & 1 & - & - \\
\hline \multirow[t]{4}{*}{ DPT } & 1 & - & - & 1 & 1 & - & - & 2 & 1 \\
\hline & 2 & 2 & 6 & 7 & 7 & 2 & 2 & - & - \\
\hline & 3 & 1 & 3 & 3 & 2 & 1 & 3 & 3 & 2 \\
\hline & Total & 3 & 9 & 11 & 10 & 3 & 5 & 5 & 3 \\
\hline
\end{tabular}

\section{Intervention III}

Twenty-three of 60 investigated tendons (38.3\%, Table 1) contained hyperechoic foci. They were present in 13/23 QFT (56.5\%), with the second most frequent localization of foci being the DPT $(34.8 \%)$. No foci were detected in two subjects.

Our results indicated that foci in the QFT were mostly located in the lateral and centro-lateral areas (76.9\%) (Table 2); $80 \%$ of the PPT bright spots were in a medial or centro-medial location, and $66.7 \%$ of those in the DPT were in centro-medial and centro-lateral locations.

Figures 2, 3 illustrate the dynamics of intratendinous foci development. The graph shows that foci occur more quickly and disappear more rapidly in the QFT than in the DPT. It also indicates that after completion of 100 jumps, a significant decline in foci occurs until the foci disappear completely at between 40 and $180 \mathrm{~min}$.

\section{DISCUSSION}

Overload of tendons is one of the main causes of degenerative tendinopathy, especially at the insertional sites (Selvanetti et al., 1997; Maffulli et al., 2003; Sharma and Maffulli, 2005; Killian et al., 2012). Insertional tendinopathy is described as a separate pathology that plays a crucial role in the diagnosis and treatment of patients (Fenwick et al., 2002; Killian et al., 2012; Nissman and Dahiya, 2014; Rees et al., 2014). It has been reported that the majority of tendon ruptures are the result of a long-lasting tendinopathy (Maffulli et al., 2003; Xu and Murrell, 2008; Rees et al., 2014; Cook et al., 2016). Our results showed that almost all intratendinous foci were observed at the tendon attachment sites. This suggests that the presence of intratendinous foci could be the initial stage of tendon damage.

Intratendinous foci formation can be explained by comparison with the intra-articular vacuum phenomenon (Coulier, 2004; Gohil et al., 2014; Yanagawa et al., 2016). Tendons (like intra-articular spaces) can be described as a separate anatomical entity with a single pressure. Therefore, excessive overload could cause a decrease in intratendinous pressure and the appearance of air bubbles. It is difficult to explain why the enthesis of the tendon can bear tensile forces four times greater than those withstood by the mid-portion (Wang, 2006), but it seems that a larger workload is needed for intratendinous air formation. On the well-known stress-strain curve, the slope of the linear region is referred to as the Young's modulus of the tendon (Wang, 2006). Microscopic damage to the tendon appears when tendons are stretched by more than $4 \%$, while macroscopic failure occurs with stretching over $8-10 \%$. It is difficult to determine the level of stretching that would result in the occurrence of intratendinous air. Nevertheless, it seems that stretching during eccentric exercise could be related to tendinopathy, and intratendinous air appears to be one of the first ultrasound signs detecting the sensitivity of tendons to overload. 


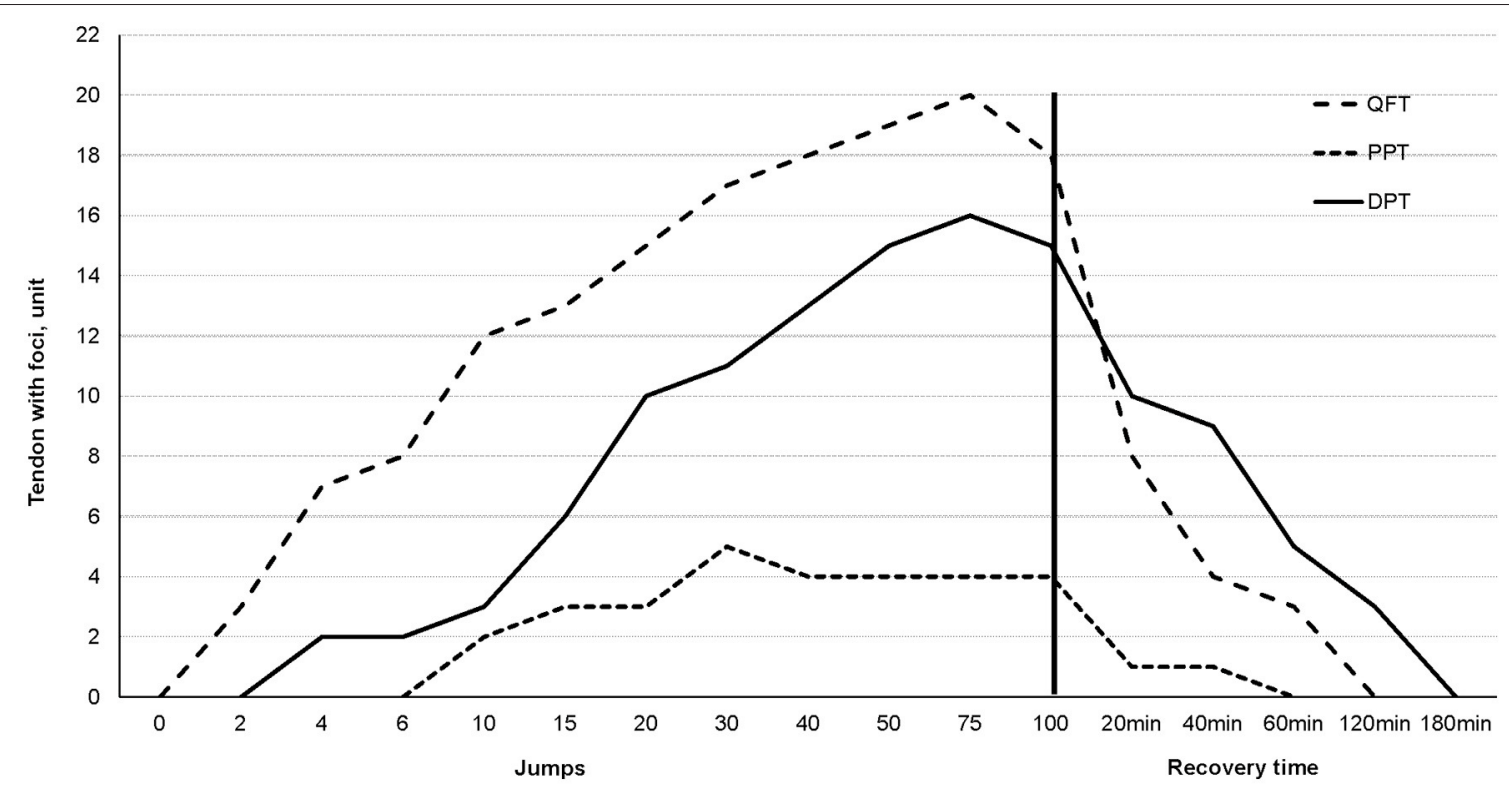

FIGURE 2 | A diagram representing the expression of intratendinous foci during the third intervention. The vertical black line indicates the end of the exercise and the beginning of relaxation.

Our observations support other evidence that this phenomenon is really not an artifact, but the presence of intratendinous air. Firstly, the foci have the characteristic features of air (blurred contours, no acoustic shadow and a down-ring artifact in ultrasound) (Figures 4A,B). Secondly, the size, form and location of intratendinous air appear to be very dynamic and dependent on the workload at the tendon insertions. Thirdly, on repeated ultrasound scan the hyperechoic foci totally disappear. And fourthly-the same features of air accumulation we have observed in several participants with different ultrasound machines-Mindray and Toshiba (Figure 1A).

We observed formation of separate sub-millimeter-sized air foci in some subjects even after only two drop jumps in QFT (Figures 2, 3.) Further jumping caused the increase in number of foci in a localized cloud-like collection. It was still possible to discern separate air bubbles, but the majority of the bubbles have already been merged into a single larger focus. The collection of air bubbles stabilizes between thirty-seventy five jumps, depending on the tendon. Interesting to note, that in some subjects the commencement of a decrease in air foci in QFT and PPT occur while an intensive workload is still present. The dissipation of air bubbles continued after the exercise quite rapidly. As observed from our experiments, the time for air disappearance from the tendons can be as fast as 20 to $180 \mathrm{~min}$, and no air bubbles were visible $24 \mathrm{~h}$ after exercise.

Intratendinous air can be multi-local and was observed not only in the DPT (Figure 1), QFT (Figure 3) and PPT (Figure 4A) but also in other tendons (unpublished data), e.g., the iliotibial band (Figure 4B), semimembranosus tendon (Figure 4C) and the biceps femoris tendon (Figure 4D). It also appears that intratendinous air may be found in other major tendons including the Achilles and plantar fascia tendons, and it is also possible that there is intraligamentous air. Differential diagnosis of air bubbles could be the insertional calcific tendinopathy and enthesophytes. The latter changes usually present with acoustic shadow and are stable during long time observation.

In the present study, we observed the typical signs of exercise-induced muscle damage after drop jumps: a prolonged decrease in MVC (within 24h) and a large increase in plasma CK activity (>10 times). It is well established that eccentric (lengthening) contraction loads can partially damage the structures of sarcomeres, myofibrils or other cytoskeletal proteins and the sarcolemma and disrupt $\mathrm{Ca}^{2+}$ regulation in muscle fibers (Lauritzen et al., 2009). The drop-jump exercise is even more demanding because the lengthening of actively contracting muscle fibers is followed immediately by a concentric contraction (Komi, 2000). Because such contractions are high force and high strain, they may cause damage not only to the muscle but also to the tendons and ligaments. It is no surprise that intratendinous air was more frequently found after this type of exercise than after maximum-intensity cycling-induced metabolic fatigue identified by an increase in blood lactate $(>12$ times) and a large decline in cycling peak power (about 70\%). The latter exercise induces metabolic perturbations such as decreased ATP and PCr and accumulation of ADP, AMP, $\mathrm{Pi}, \mathrm{H}^{+}$, and $\mathrm{Mg}^{2+}$ and negatively affects the cross-bridge interaction and calcium shuttling between the sarcoplasmic reticulum and the myoplasm (Ament and Verkerke, 2009), but is much less mechanically demanding in respect of muscle lengthening. We also made the assumption that such a high number of drop jumps might induce signs of pathological changes to the tendons, but we observed only minor pathological changes on ultrasonography, which had disappeared after $24 \mathrm{~h}$. 


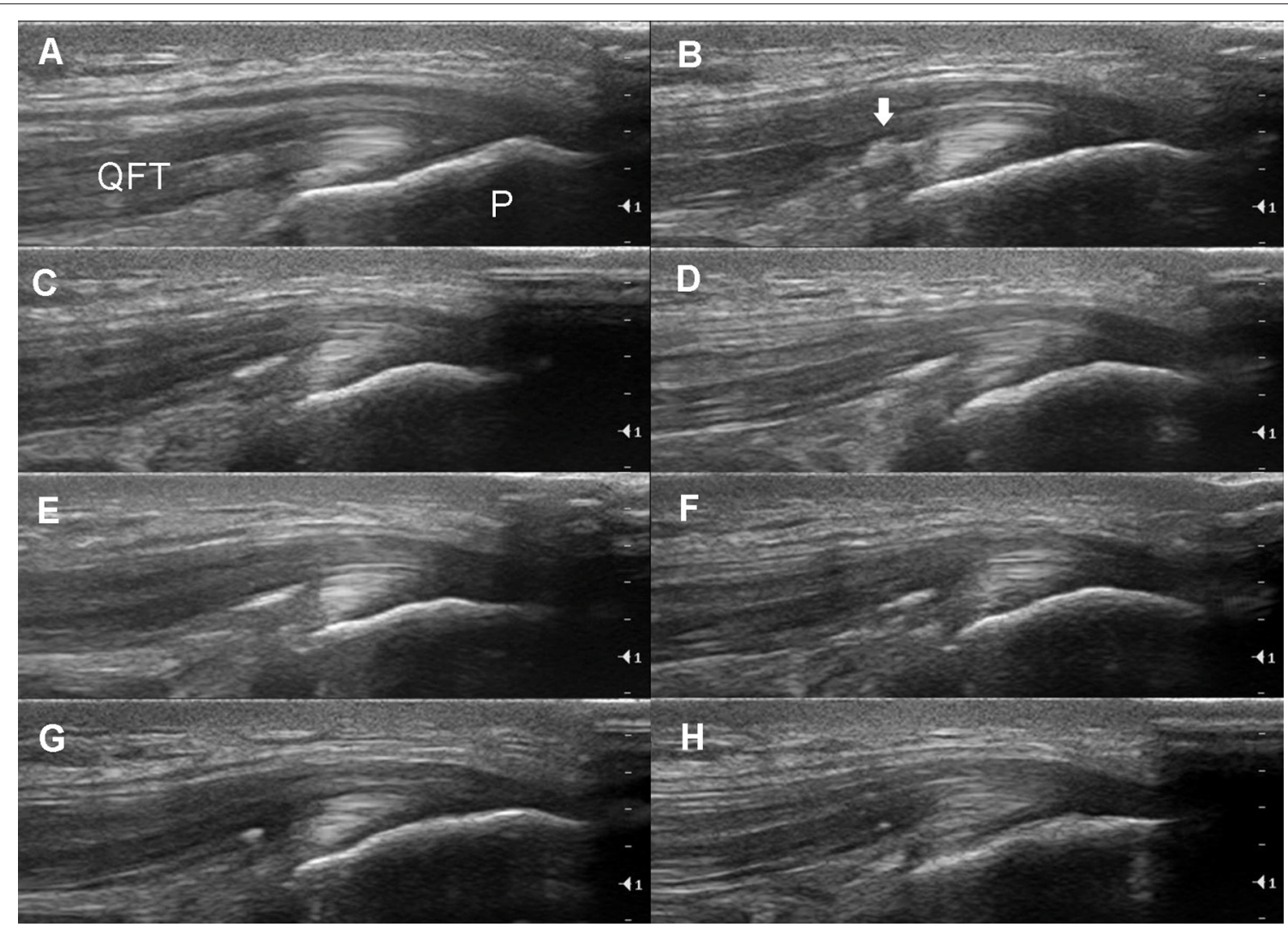

FIGURE 3 | Longitudinal ultrasound imaging of the same subject. (A) The normal right quadriceps femoris tendon (QFT) and its insertion into the patella (P).

(B) Intratendinous collections of bright foci (arrow) can already be clearly visualized after two jumps. Dynamic changes in the intratendinous foci can be seen after 15 (C), 40 (D), 75 (E), and 100 (F) jumps and $20 \mathrm{~min}$ (G) and $40 \mathrm{~min}$ (H) after exercise.



FIGURE 4 | Various attachments of the knee tendons showing signs of intratendinous air (white arrows). (A) The insertion of the proximal patellar tendon (PPT) into the patella (P). The small hyperechogenic focus (arrow) appears with blurred contours and without any acoustic shadow. (B) The long axis of the insertion of the iliotibial band (ITB). The arrow shows the collection of the intratendinous air with a down-ring artifact (star) (Video 4b). GT, Gerdy's tubercle; LM, lateral meniscus. (C) The long axis of semimembranosus tendon (ST) insertion into the posterior aspect of the medial condyle of tibia (T). (D) The common insertion of the biceps femoris tendon (BFT) and lateral collateral ligament (not shown) in the fibular head (FH). 
One of the weaknesses of our study is that the subjects underwent only ultrasound measurements in addition to standard $12 \mathrm{MHz}$ ultrasound device does not allow estimating air bubbles' size accurately. However, it is rather difficult to evaluate such small air accumulations in the tendons using other imaging techniques. We did not estimate air bubbles size consistently but random assessment found that the size of the smallest air bubble was about 190-270 $\mu \mathrm{m}$. Obviously, during radiographic or CT imaging it is difficult to differentiate such small air bubbles because they overlap with the surrounding tissues. During MRI, the air bubbles are too small to cause susceptibility artifacts, and in addition, the tendon itself appears black, as do the air bubbles (Malghem et al., 2011; Hodgson et al., 2012). Further research is planned to better characterize intratendinous air by obtaining higher quality images.

\section{CONCLUSIONS}

The presence of intratendinous air seems related to high-magnitude high-force high-strain exercise at particular tendon areas. It may represent the tendon stress response to overload conditions. Further research is required to establish the possible links between the presence of intratendinous air and the development of tendinopathy, especially for individuals involved in regular eccentric-type exercise.

\section{REFERENCES}

Ament, W., and Verkerke, G. J. (2009). Exercise and fatigue. Sports. Med. 39, 389-422. doi: 10.2165/00007256-200939050-00005

Cook, J. L., Rio, E., Purdam, C. R., and Docking, S. I. (2016). Revisiting the continuum model of tendon pathology: what is its merit in clinical practice and research? Br. J. Sports Med. 50, 1187-1191. doi: 10.1136/bjsports-2015095422

Coulier, B. (2004). The spectrum of vacuum phenomenon and gas in spine. JBR-BTR. 87, 9-16.

D’Anastasi, M., Birkenmaier, C., Schmidt, G. P., Wegener, B., Reiser, M. F., and Baur-Melnyk, A. (2011). Correlation between vacuum phenomenon on CT and fluid on MRI in degenerative disks. Am. J. Roentgenol. 197, 1182-1189. doi: 10.2214/AJR.10.6359

Fairbairn, K. J., Mulligan, M. E., Murphey, M. D., and Resnik, C. S. (1995). Gas bubbles in the hip joint on CT: an indication of recent dislocation. Am. J. Roentgenol. 164, 931-934. doi: 10.2214/ajr.164.4.7726051

Feng, S. W., Chang, M. C., Wu, H. T., Yu, J. K., Wang, S. T., and Liu, C. L. (2011). Are intravertebral vacuum phenomena benign lesions? Eur. Spine. J. 20, 1341-1348. doi: 10.1007/s00586-011-1789-3

Fenwick, S. A., Hazleman, B. L., and Riley, G. P. (2002). The vasculature and its role in the damaged and healing tendon. Arthritis Res. 4, 252-260. doi: $10.1186 /$ ar416

Fick, R. (1919). Handbuch der Anatomie und Mechanik der Gelenke. Jena: Gustave Fischer.

Gohil, I., Vilensky, J., and Weber, E. (2014). Vacuum phenomenon: clinical relevance. Clin. Anat. 27, 455-462. doi: 10.1002/ca.22334

Hodgson, R., O'Connor, P., and Grainger, A. (2012). Tendon and ligament imaging. Br. J. Radiol. 85, 1157-1172. doi: 10.1259/bjr/34786470

Ito, H., Yoshikawa, T., Hayashi, N., and Ohtomo, K. (2008). MDCT demonstration of intraarticular gas in the glenohumeral joint and sternoclavicular joint with reference to arm position. Radiat. Med. 26, 422-426. doi: 10.1007/s11604-008-0253-8

\section{PATIENT CONSENT}

Informed consent was obtained from all individual subjects included in the study.

\section{ETHICS STATEMENT}

This study was carried out in accordance with the recommendations of Kaunas regional biomedical research ethics committee with written informed consent from all subjects. All subjects gave written informed consent in accordance with the Declaration of Helsinki. The protocol was approved by the Kaunas regional biomedical research ethics committee.

\section{AUTHOR CONTRIBUTIONS}

SR, ASk, and SK contributed to the conception, design, analysis and interpretation of the work. VP, DS, MB, ASn, NB, and RR contributed to the acquisition, analysis and interpretation of the data. All authors contributed to the drafting of the manuscript and approved the final version.

\section{ACKNOWLEDGMENTS}

The authors would like to acknowledge the help of all the people involved in this project and, more specifically, to the subjects.

Jordanov, M., and Block, J. (2010). Minute amounts of intraarticular gas mimicking torn discoid lateral menisci. J. Magn. Reson. Imaging 31, 698-702. doi: 10.1002/jmri.22082

Kamandulis, S., Snieckus, A., Venckunas, T., Aagaard, P., Masiulis, N., and Skurvydas, S. (2012). Rapid increase in training load affects markers of skeletal muscle damage and mechanical performance. J. Strength. Cond. Res. 26, 2953-2961. doi: 10.1519/JSC.0b013e318243ff21

Killian, M. L., Cavinatto, L., Galatz, L. M., and Thomopoulos, S. (2012). The role of mechanobiology in tendon healing. J. Shoulder. Elbow. Surg. 21, 228-237. doi: 10.1016/j.jse.2011.11.002

Komi, P. V. (2000). Stretch-shortening cycle: a powerful model to study normal and fatigued muscle. J. Biomech. 33, 1197-1206. doi: 10.1016/S0021-9290(00)00064-6

Lauritzen, F.,., Paulsen, G., Raastad, T., Bergersen, L. H., and Owe, S. G. (2009). Gross ultrastructural changes and necrotic fiber segments in elbow flexor muscles after maximal voluntary eccentric action in humans. J. Appl. Physiol. 107, 1923-1934. doi: 10.1152/japplphysiol.00148. 2009

Liu, Z., Yan, W., and Zhang, L. (2015). Analysis of the vacuum phenomenon in plain hip radiographs in children. Int. J. Clin. Exp. Med. 8, 3325-3331.

Maffulli, N., Wong, J., and Almekinders, L. (2003). Types and epidemiology of tendinopathy. Clin. Sports. Med. 22, 675-692. doi: 10.1016/S0278-5919(03)00004-8

Malghem, J., Omoumi, P., Lecouvet, F. E., and Vande Berg, B. C. (2011). Presumed intraarticular gas microbubbles resulting from a vacuum phenomenon: visualization with ultrasonography as hyperechoic microfoci. Skeletal Radiol. 40, 1287-1293. doi: 10.1007/s00256-011-1107-5

Moncada, G., Oyarzo, J. F., Moncada, M., Marholz, C., and Millas, R. (2008). Vacuum phenomenon in the temporomandibular joint: a clinical, computed tomography and magnetic resonance case report. Dentomaxillofac. Radiol. 37, 236-239. doi: 10.1259/dmfr/12509397

Motamedi, D., Everist, B. M., Mahanty, S. R., and Steinbach, L. S. (2014). Pitfalls in shoulder MRI: part 2Biceps tendon, bursae and cysts, incidental 
and postsurgical findings, and artifacts. Am. J. Roentgenol. 203, 508-515. doi: 10.2214/AJR.14.12849

Nissman, D. B., and Dahiya, N. (2014). Ultrasonography of Tendons. Ultrasound Clin. 9, 489-512. doi: 10.1016/j.cult.2014.03.001

Rees, J., Stride, M., and Scott, A. (2014). Tendons time to revisit inflammation. $\mathrm{Br}$. J. Sports. Med. 48, 1553-1557. doi: 10.1136/bjsports-2012-091957

Schröder, J. H., Marschalek, N., Hufeland, M., and Perka, C. (2016). The 'Hip Vacuum Sign, a new radiographic phenomenon in femoro-acetabular impingement. J. Hip. Preserv. Surg. 3, 346-351. doi: 10.1093/jhps/hnw018

Selvanetti, A., Cipolla, A., and Puddu, G. (1997). Overuse tendon injuries: Basic science and classification. Oper. Tech. Sports. Med. 5, 110-117. doi: 10.1016/S1060-1872(97)80031-7

Sharma, P, and Maffulli, N. (2005). Tendon injury and tendinopathy: healing and repair. J. Bone. Joint. Surg. Am. 87, 187-202. doi: 10.2106/00004623-200501000-00030

Stallenberg, B., A., Madani, Burny, F., and Gevenois, P. A. (2001). The vacuum phenomenon: a ct sign of nonunited fracture. Am. J. Roentgenol. 176, 1161-1164. doi: 10.2214/ajr.176.5.1761161

Wadhwa, V., Cho, G., Moore, D., Pezeshk, P., Coyner, K., and Chhabra, A. (2016). T2 black lesions on routine knee MRI: differential considerations. Eur. Radiol. 26, 2387-2399. doi: 10.1007/s00330-015-4027-2
Wang, J. H. (2006). Mechanobiology of tendon. J. Biomech. 39, 1563-1582. doi: 10.1016/j.jbiomech.2005.05.011

$\mathrm{Xu}$, Y., and Murrell, G. A. C. (2008). The basic science of tendinopathy. Clin. Orthop. Relat. Res. 466, 1528-1538. doi: 10.1007/s11999-0080286-4

Yanagawa, Y., Ohsaka, H., Jitsuiki, K., Yoshizawa, K., Takeuchi, I., Omori, K., et al. (2016). Vacuum phenomenon. Emerg. Radiol. 23, 377-382. doi: 10.1007/s10140-016-1401-6

Conflict of Interest Statement: The authors declare that the research was conducted in the absence of any commercial or financial relationships that could be construed as a potential conflict of interest.

Copyright (C) 2017 Rutkauskas, Paleckis, Skurvydas, Satkunskiene, Brazaitis, Snieckus, Baranauskiene, Rancevas and Kamandulis. This is an open-access article distributed under the terms of the Creative Commons Attribution License (CC BY). The use, distribution or reproduction in other forums is permitted, provided the original author(s) or licensor are credited and that the original publication in this journal is cited, in accordance with accepted academic practice. No use, distribution or reproduction is permitted which does not comply with these terms. 